\title{
Does melatonin help save dopaminergic cells in MPTP-treated mice?
}

\author{
Jeannine Ma ${ }^{\mathrm{b}}$, Victoria E. Shaw ${ }^{\mathrm{c}}$, John Mitrofanis ${ }^{\mathrm{a}, \mathrm{b}, \mathrm{c}, *}$ \\ ${ }^{a}$ Medical School, Australian National University, Australia \\ ${ }^{\mathrm{b}}$ CNS Stability and Degeneration, Research School of Biological Sciences, Australian National University, Australia \\ ${ }^{\mathrm{c}}$ Discipline of Anatomy \&' Histology F13, University of Sydney, Sydney, NSW 2006, Australia
}

\section{A R T I C L E I N F O}

\section{Article history:}

Received 7 June 2008

Received in revised form 23 July 2008

Accepted 29 July 2008

\section{Keywords:}

Basal ganglia

Cell death

Neuroprotection

Parkinson disease

\begin{abstract}
A B S T R A C T
This study explores whether melatonin neuroprotects dopaminergic cells of the substantia nigra pars compacta (SNc) from degeneration in 1-methyl-4-phenyl-1,2,3,6-tetrahydropyridine (MPTP)-treated mice (well-known animal model of Parkinson disease). BALB/c albino mice were divided into four experimental groups. In each, mice received three series (over a 24-h period) of two intraperitoneal injections ( $1 \mathrm{~h}$ apart) in different combinations. The different groups and their combinations of injections were: (1) Saline (saline, saline); (2) Mel (melatonin, saline); (3) MPTP (saline, MPTP); (4) Mel-MPTP (melatonin, MPTP). Six days after the last injection, all mice were perfused transcardially with aldehyde fixative. Brains were processed for routine tyrosine hydroxylase ( $\mathrm{TH}$; rate limiting enzyme for dopamine production) immunochemistry and Nissl staining. Our results - using unbiased stereology - showed that there were more $\mathrm{TH}^{+}(50 \%)$ and Nissl-stained (30\%) cells in the SNc of the Mel-MPTP group compared to the MPTP group, indicating a clear saving or neuroprotection of these cells. In fact, we found no significant difference between the number of $\mathrm{TH}^{+}$and Nissl-stained SNc cells in the Mel-MPTP group compared to the controls, namely Saline and Mel groups. This indicated that melatonin pre-treatment potentially neuroprotected all the SNc cells from MPTP toxicity and death.
\end{abstract}

Crown Copyright $\odot 2008$ Published by Elsevier Ltd. All rights reserved.

\section{Introduction}

Parkinson disease is characterised by a loss of dopaminergic cells within the SNc of the midbrain [1]. This cell loss results in a reduction of dopamine levels in the striatum, which in turn, triggers a cascade of abnormal neural circuits that manifest in the distinct signs of Parkinson disease, namely lead-pipe rigidity, akinesia and tremor [2]. The factors that generate the initial loss of SNc dopaminergic cells are not entirely clear, but there is evidence for mitochondrial dysfunction as a result of exposure to an environmental toxin [2].

The first line treatment for patients with Parkinson disease is dopamine-replacement drug therapy. Perhaps the best known example of this therapy is Levodopa, which remains the "gold standard". However, the use of Levodopa - as well as many other

Abbreviations: 6OHDA, 6-hydroxydopamine; CoQ10, co enzyme Q 10; cp, cerebral peduncle; $\mathrm{CPu}$, caudate-putamen complex; GDNF, glial cell line derivative neurotrophic factor; ic, internal capsule; Mel, melatonin; MPTP, 1-methyl-4-phenyl1,2,3,6-tetrahydropyridine; PaG, periaqueductal grey matter; PBS, phosphate buffered saline; Pt, pretectum; SCol, superior colliculus; SNc, substantia nigra pars compacta; SNr, substantia nigra pars reticulata; $\mathrm{TH}$, tyrosine hydroxylase; VTA, ventral tegmental area; ZI, zona incerta.

* Corresponding author. Discipline of Anatomy \& Histology F13, University of Sydney, Sydney, NSW 2006, Australia. Tel.: +612 93512500.

E-mail address: zorba@med.usyd.edu.au (J. Mitrofanis). drugs - has been shown to have its problems. For instance, longterm use leads to major motor side-effects and decreased effectiveness [3]. Further, and perhaps most importantly, such treatment is largely symptomatic and does not stop the pathology $[3,4]$. For this reason, there is a need to develop new treatments aimed at stopping or slowing down the progression of the disease. Indeed, there have been many studies over the years - mainly in animal models of the disease - reporting that some substances, or treatments (e.g., deep brain stimulation [4]), save or offer neuroprotection to the dopaminergic system in parkinsonian cases. One such substance is the indoleamine, melatonin.

Melatonin is released naturally into the blood stream mainly by the pineal gland, but also by other structures such as the retina, bone marrow and gastrointestinal system. The classical function associated with melatonin is entraining body circadian rhythms, an internal 24-h time-keeping system [5]. In more recent years, melatonin has been shown to also have an anti-oxidant function that protects cells from oxygen free radical damage [6]. In particular, melatonin has been shown to either stimulate radical detoxifying enzymes or scavenge hydroxyl radicals and offer protection against oxidative stress [7].

In this study, we have explored the cell saving or neuroprotective effects of melatonin in MPTP-treated mice. There has been few previous studies that have reported on the impact of melatonin on the morphology and number of dopaminergic cells in 
the SNc of parkinsonian animals [8,9]; previous studies have focussed mainly on changes in activity or dopamine levels in the striatum [10-17] or in the SNc [18]. In each of these studies, melatonin was administered either just prior to or immediately after parkinsonian insult (either 6-hydroxydopamine (6OHDA) lesion or MPTP treatment). In this study, we have followed a similar paradigm and combination of melatonin administration and parkinsonian insult. This paradigm is somewhat unlike the clinical reality where SNc cell loss occurs prior to therapeutic intervention. However, in our experimental study - as with the above mentioned previous ones - we hoped to explore the maximum effect of melatonin treatment on the number of SNc cells and hence determine more systematically its neuroprotective effects.

\section{Materials and methods}

2.1. Subjects

Male albino BALB/c mice ( $\sim 20 \mathrm{~g} ; \sim 8$ weeks old; $n=53$ ) were used. Animals were housed in a $12 \mathrm{~h} \mathrm{light/dark} \mathrm{cycle} \mathrm{with} \mathrm{unlimited} \mathrm{access} \mathrm{to} \mathrm{food} \mathrm{and} \mathrm{water.} \mathrm{All}$ experiments were approved by the Animal Ethics Committee of the Australian National University and University of Sydney.

\subsection{Experimental design}

There were four experimental groups in this study (see Fig. 1). In each, mice received three series (over a 24 -h period) of two intraperitoneal injections ( $1 \mathrm{~h}$ apart) in different combinations. The different groups and their combinations of injections were;

(1) Saline group ( $n=11)$ : injections of saline ( $5 \%$ ethanol) followed by saline.

(2) Melatonin (Mel) group $(n=10)$ : injections of melatonin $(20 \mathrm{mg} / \mathrm{kg}$, in $5 \%$ ethanol/saline) followed by saline.

(3) MPTP group $(n=18)$ : injections of saline (5\% ethanol) followed by MPTP $(20 \mathrm{mg} / \mathrm{kg}$ in saline).

(4) Mel-MPTP group $(n=14)$ : injections of melatonin $(20 \mathrm{mg} / \mathrm{kg})$ followed by MPTP $(20 \mathrm{mg} / \mathrm{kg})$.

The quantities of melatonin and MPTP injected were within the range used by previous studies [8-18]. It should be noted that these studies have used different means of administrating melatonin, either via drinking water $[11,12]$, by subcutaneous mini-pumps [11] or by intraperitoneal injection [8-10,13-16]. In this study, we chose the more quantifiable route of intraperitoneal injection. For the MPTP doses, we used a milder dose of $60 \mathrm{mg} / \mathrm{kg}(3 \times 20 \mathrm{mg} / \mathrm{kg}$ injections $)$ that results in a less severe loss of SNc cells. This allows for more time for therapeutic intervention and greater sign of possible neuroprotection [4].

After the last series of injections, mice were allowed to survive for 6 days; this survival period is sufficient for MPTP to impart toxicity among dopaminergic cells [4]. After each injection, particularly after the MPTP ones, the mice were monitored closely for any changes in behaviour.

\subsection{Histology and immunohistochemistry}

After the survival period was complete, mice were anaesthetised after an intraperitoneal injection of sodium pentobarbital $(60 \mathrm{mg} / \mathrm{ml})$. They were then perfused transcardially with $0.1 \mathrm{M}$ phosphate-buffered saline (PBS; pH 7.4), followed by $4 \%$ buffered formaldehyde. The brains were removed and post-fixed overnight in the same solution. Next, brains were placed in PBS with the addition of $30 \%$ sucrose until the block sank. The forebrain and midbrain were then sectioned coronally and serially (at $55 \mu \mathrm{m}$ thickness) using a freezing microtome. All sections were collected in PBS. One series of sections (1:2) was mounted onto gelatinised slides and then stained for Nissl (neutral red) using routine procedures. The other series were stored in PBS and used for immunohistochemistry. For this, sections were immersed in a solution of $0.1 \%$ Triton (Sigma) and 10\% normal goat serum in PBS at room temperature for $1 \mathrm{~h}$. Sections were incubated in anti-TH (Chemicon; $1: 500)$ for $24 \mathrm{~h}$. Next, sections were incubated in biotinylated anti-mouse IgG (Sigma; $1: 200$ ) for $2 \mathrm{~h}$ at room temperature. Finally, sections were incubated in Extravidin-peroxidase complex (Sigma; 1:200) at room temperature for $1 \mathrm{~h}$. To visualise the bound antibody, sections were reacted in nickel-Tris-buffered saline ( $\mathrm{pH}$ 7.4)-3,3'-diaminobenzidine tetrahydrochloride (Sigma) solution. In between each incubation, sections were washed in three changes of PBS. Sections were mounted onto gelatinised slides, air dried overnight, dehydrated in ascending alcohols, cleared in Histoclear and coverslipped using DPX. Some immunostained sections were counterstained lightly with Neutral Red as well. For controls, sections were processed as described above, except that there was no primary antibody used. Control sections were immunonegative.

\subsection{Analysis}

The total number of cells in the SNc was estimated using routine stereological methods. Counts were made from the SNc (right hand side), the main region of compact cells that has been shown to undergo the most degeneration after parkinsonian insult [1,4]. This region was distinguished from the smaller cells of the ventral tegmental area (VTA) that were located more medial [4,19].

In the SNc, cell number was analysed using $\mathrm{TH}$ immunoreactivity and Nissl staining. These two methods were used to explore whether the loss of cells after MPTP treatment was due to loss of antigen expression (TH) or to a loss of the cells themselves (Nissl); in other words, we explored whether there was functional and/ or true neuroprotection. TH immunohistochemistry labels "healthy" cells that express the dopamine phenotype, that is, this labelling provides an index of function of dopaminergic cells. On the other hand, Nissl staining labels all cells regardless of phenotypic expression [4].

Following procedures as outlined by previous studies, the outline of the SNc (from $\mathrm{TH}$-immunostained sections that defines the nucleus most distinctly) was drawn and the volume of the region was calculated using Cavalieri's formula. The estimated total number of cells within the SNc was obtained using the optical fractionator method [4]. Systematic random sampling of locations (with an unbiased counting frame) within the defined SNc boundaries of every 2nd section was undertaken (by two observers, blind to treatment). All cells (nucleated only) that came into focus within an unbiased virtual counting space were counted. As a general rule, the number of cells and volumes (mean of two observer counts) were expressed as the mean \pm standard error.

For comparisons between groups (using the StatGraphPad programme), a oneway ANOVA test ( $F$ test; $p$ value) was performed with a Tukey multiple comparison test ( $q$ test; $p$ value). Schematic diagrams and digital images were constructed using Adobe Photoshop and Microsoft PowerPoint programmes.

\section{Results}

The results that follow are presented in five main parts: topography of dopaminergic cells, MPTP toxicity, morphology of dopaminergic cells, volume of the SNc and number of dopaminergic cells.

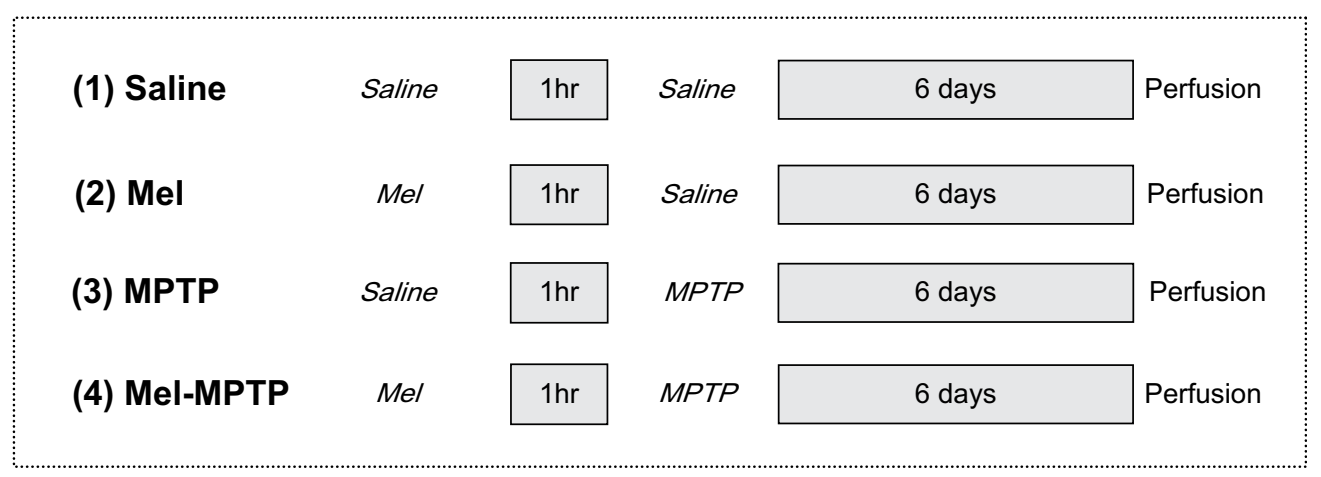

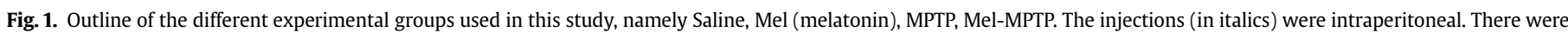

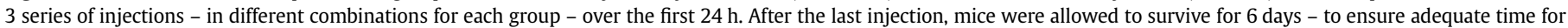
toxicity of MPTP - before being perfused and prepared for immunocytochemistry and histology. 


\subsection{Topography of dopaminergic cells}

The SNc was identified as the compact group of cells located immediately superior to the substantia nigra pars reticulata $(\mathrm{SNr})$, and lateral to the smaller cells of the VTA $[4,19]$. Fig. 2A shows schematic diagrams of the brainstem sections matching closely to those used to count the number of SNc cells. Fig. 2B shows a THimmunostained section indicating the boundaries of the SNc which was used to measure area and subsequently volume and cell number. In our analysis, we counted only the $\mathrm{TH}^{+}$and Nissl-stained cells that had a nucleus (arrows Fig. 2C,D); non-nucleated cells were not counted (arrowheads Fig. 2C,D). Such a method ensured consistency in our approach, even though our counts of every second section made it unlikely that we would count the same cell twice [4].

\subsection{MPTP toxicity}

The success of our MPTP treatment was measured in two ways. First, we examined the patterns of $\mathrm{TH}$ immunoreactivity in the midbrain of each of the groups used in this study. Fig. 3 shows

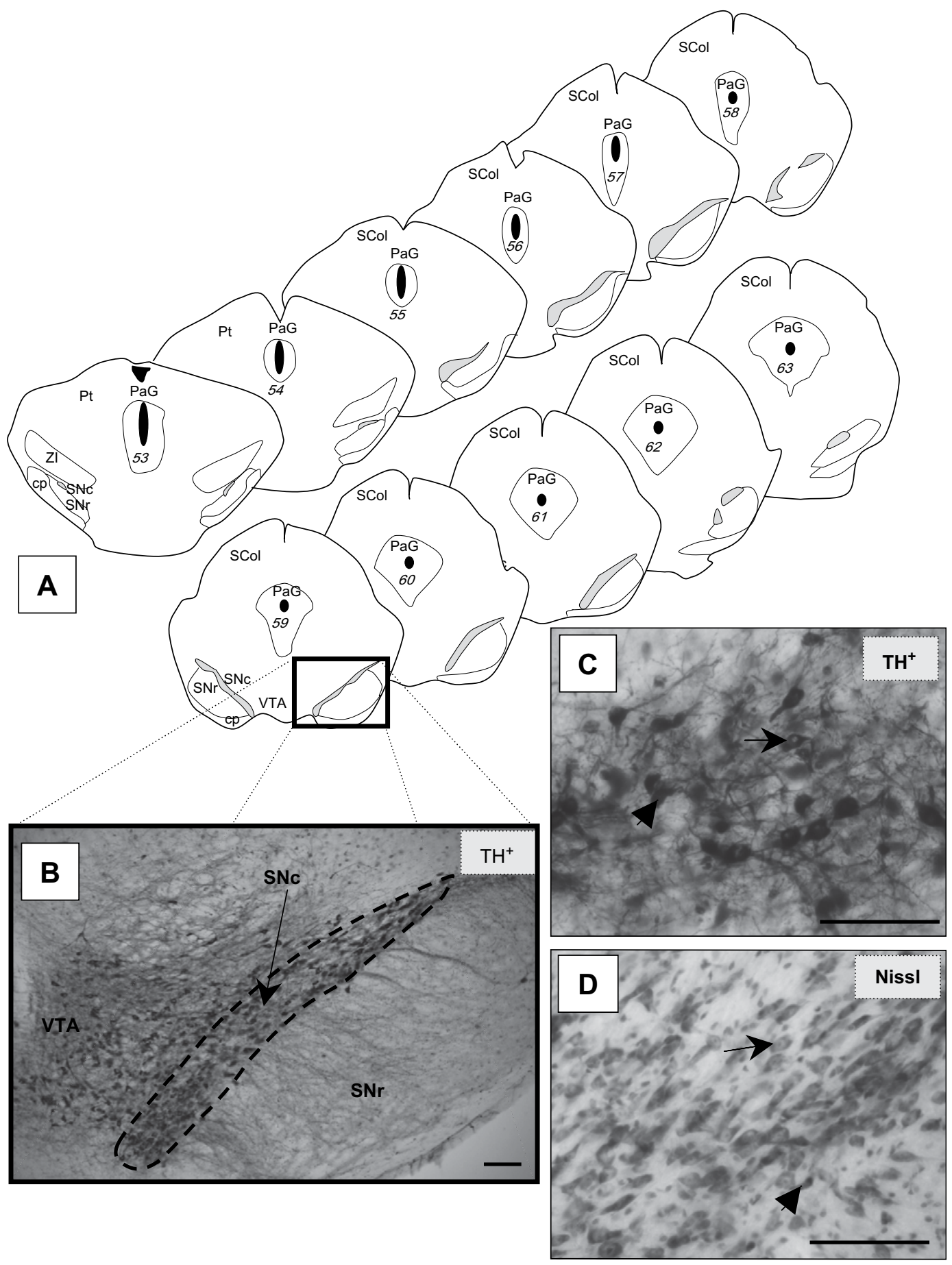

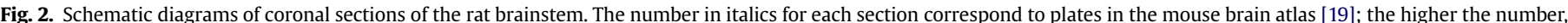

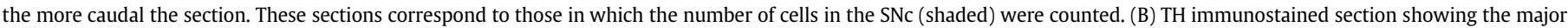

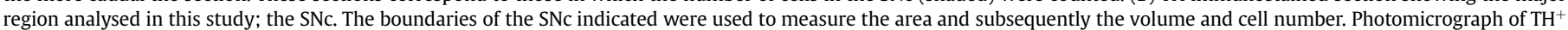

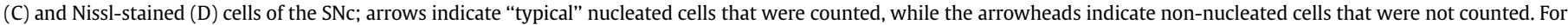
(B)-(D) dorsal to top, lateral to right. Scale bar $=100 \mu \mathrm{m}$. 
TH-immunostained midbrain sections from the Saline (Fig. 3A), Mel (Fig. 3B), MPTP (Fig. 3C) and Mel-MPTP (Fig. 3D) groups. There were fewer $\mathrm{TH}^{+}$cells in the SNc of the MPTP group compared to all the other groups, namely Saline, Mel and MelMPTP. A more quantitative analysis of the differences in $\mathrm{TH}^{+}$ (+Nissl-stained) cell number in the different groups is considered later in this manuscript. Second, we examined the patterns of $\mathrm{TH}^{+}$ terminal labelling in the $\mathrm{CPu}$. In the MPTP group, we observed fewer $\mathrm{TH}^{+}$terminals in the $\mathrm{CPu}$ (Fig. 3E), when compared to the Saline, Mel and Mel-MPTP (Fig. 3F) groups.

We did not observe any major behavioural deficits in the mice after our MPTP injections, although in some instances, the mice would become quiescent immediately after injection. However, these mice soon returned to normal activity - eating and grooming within the next few hours. Following the saline and melatonin injections, no behavioural deficits were seen in any of the mice.

\subsection{Morphology of dopaminergic cells}

We examined the morphology of midbrain dopaminergic cells in the four experimental groups using TH immunoreactivity and Nissl staining. In general, there were no substantial differences in the overall morphology of $\mathrm{TH}^{+}$or Nissl-stained cells in each of the groups.
Fig. 4 shows typical examples of labelled cells in the SNc in each of the experimental groups, namely Saline (Fig. 4A,B), Mel (Fig. 4C,D), MPTP (Fig. 4E,F) and Mel-MPTP (Fig. 4G,H). In each case, TH immunoreactivity and Nissl staining was seen in cells with generally rounded somata; $\mathrm{TH}$ immunoreactivity also revealed immunoreactive dendrites that often formed a dense lattice work within the area.

\subsection{Volume of the SNC}

The volume of the SNc in each of the experimental groups was very similar. In the Saline group, SNc volume was $0.14 \pm 0.01 \mathrm{~mm}^{3}$, while in the Mel group, it was $0.16 \pm 0.010 .01 \mathrm{~mm}^{3}$. In the MPTP group, SNc volume was $0.14 \pm 0.010 .01 \mathrm{~mm}^{3}$, while in the MelMPTP group, it was $0.15 \pm 0.010 .01 \mathrm{~mm}^{3}$ (Fig. 5).

When comparing these values between the four experimental groups using an ANOVA test, no significant differences were found $(F= \pm 0.011 .4 ; p \pm 0.01= \pm 0.010 .26)$. A multiple comparison test also revealed no significant differences between the volumes of these groups (for each group comparison, $q \pm 0.01< \pm 0.013 .8$; $p \pm 0.01> \pm 0.010 .05)$. Thus, there was no major impact on the overall volume of the SNc after melatonin or MPTP treatment in our study.
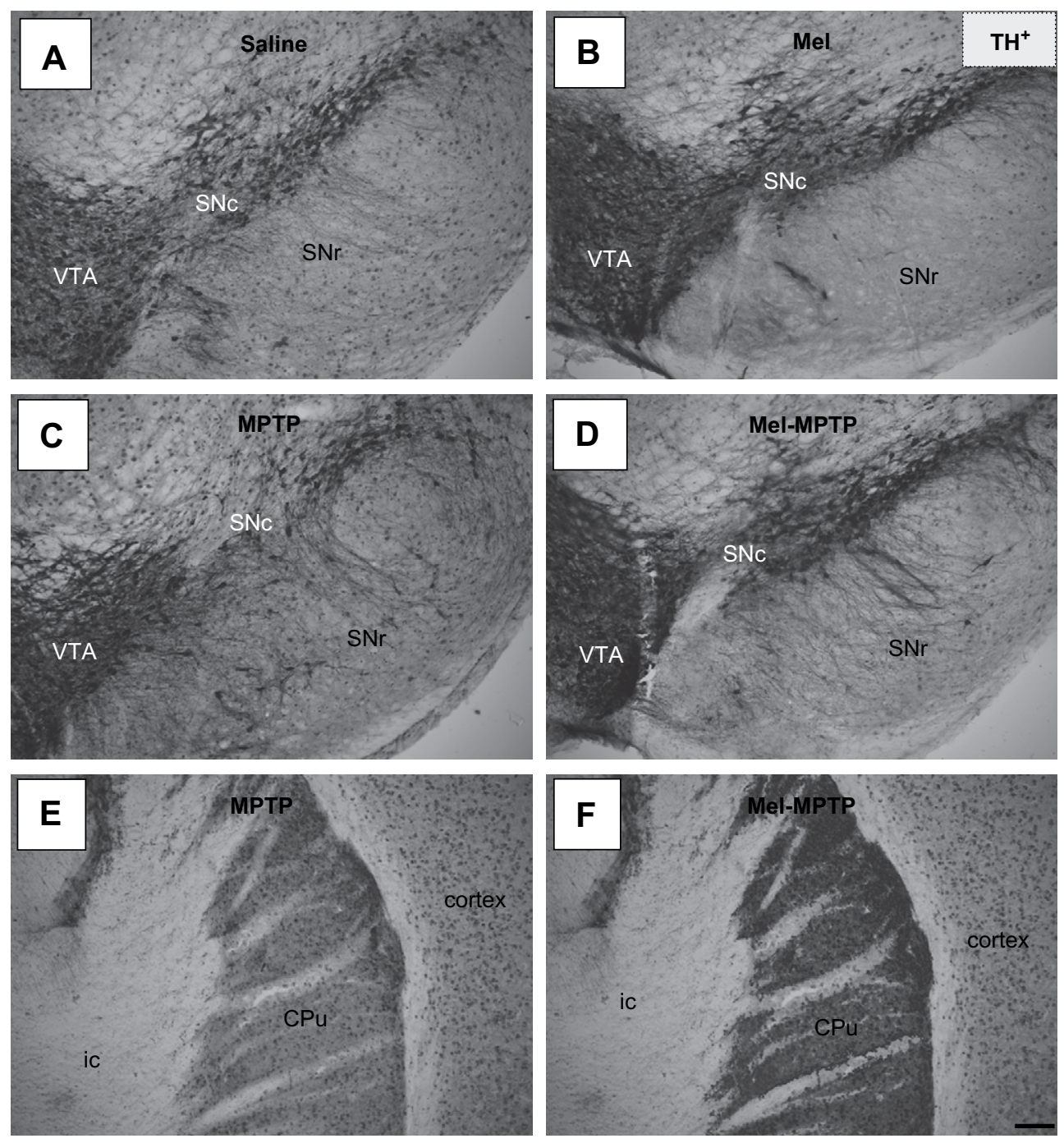

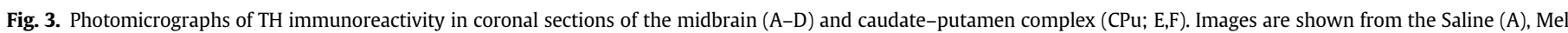

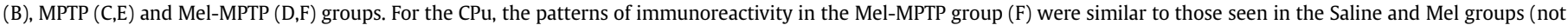
shown). Sections were immunostained for $\mathrm{TH}$ and then counterstained lightly with neutral red. For all images, dorsal to top and lateral to right. Scale bars $=100 \mu \mathrm{m}$. 

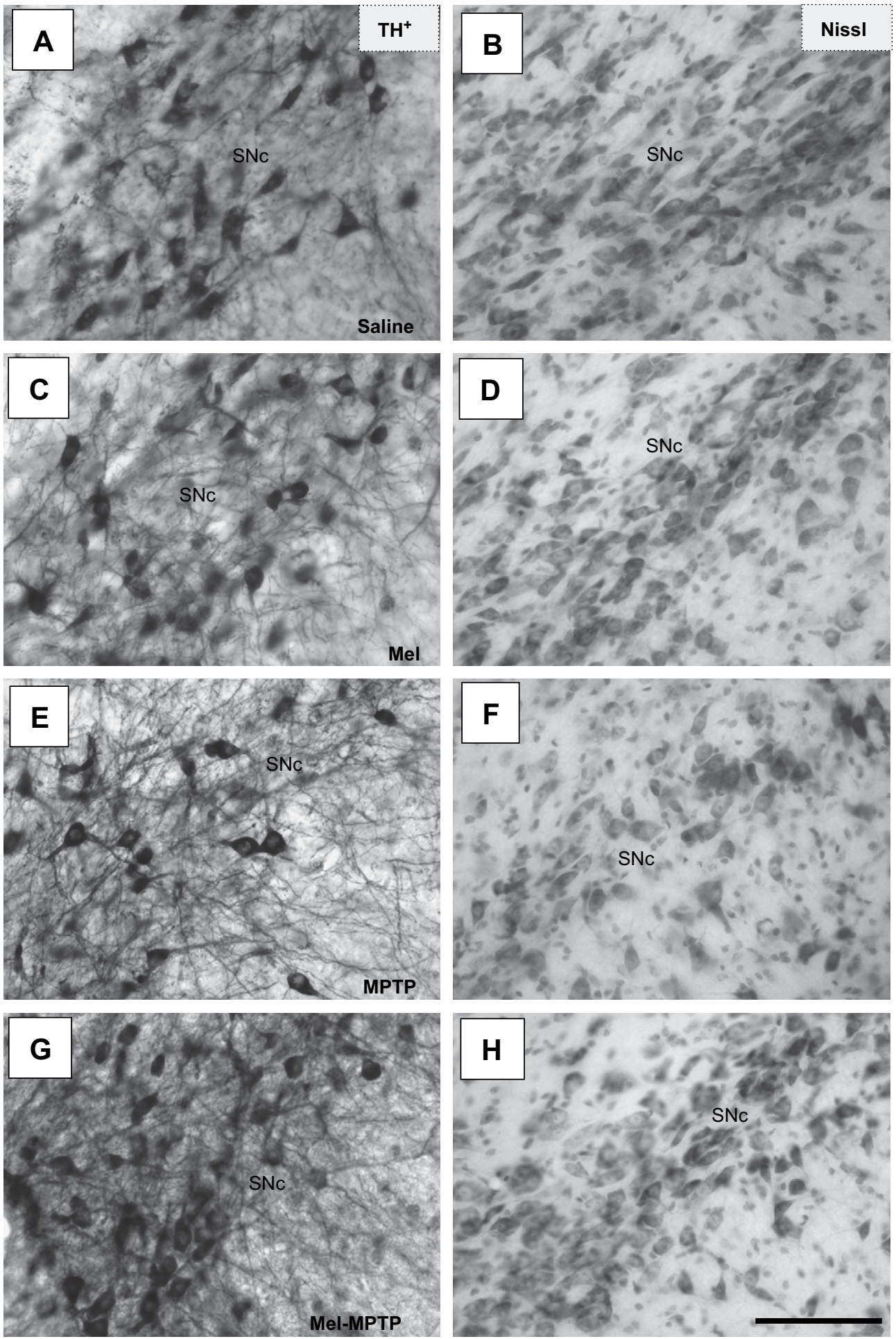

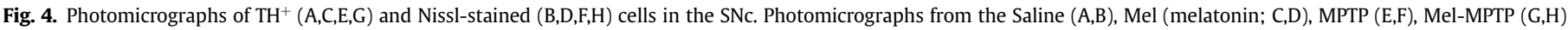

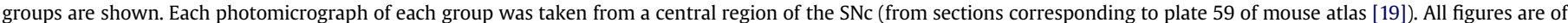
coronal sections; dorsal to top and lateral to right. Scale bar $=100 \mu \mathrm{m}$.

\subsection{Number of dopaminergic cells}

The number of $\mathrm{TH}^{+}$and Nissl-stained cells in the SNc of each of the experimental groups in this study will be considered separately below.

\subsection{1. $\mathrm{TH}^{+}$cells}

The graph in Fig. 6 shows the estimated number of $\mathrm{TH}^{+}$cells in the SNc of the four experimental groups. In the Saline and Mel groups, the total number of $\mathrm{TH}^{+}$cells was very similar, being $12,150 \pm 0.01800$ and $13,200 \pm 0.01800$ (Fig. 6). These estimates for the total number of $\mathrm{TH}^{+}$cells in the SNc of mice were within the range reported by previous studies [20].

In the MPTP group, the number of $\mathrm{TH}^{+}$cells was $6250 \pm 0.01550$, indicating a 50\% reduction in SNc cell number from the Saline and Mel groups. For the Mel-MPTP group, the number of $\mathrm{TH}^{+}$cells was $12,350 \pm 0.01850$, which was similar to 


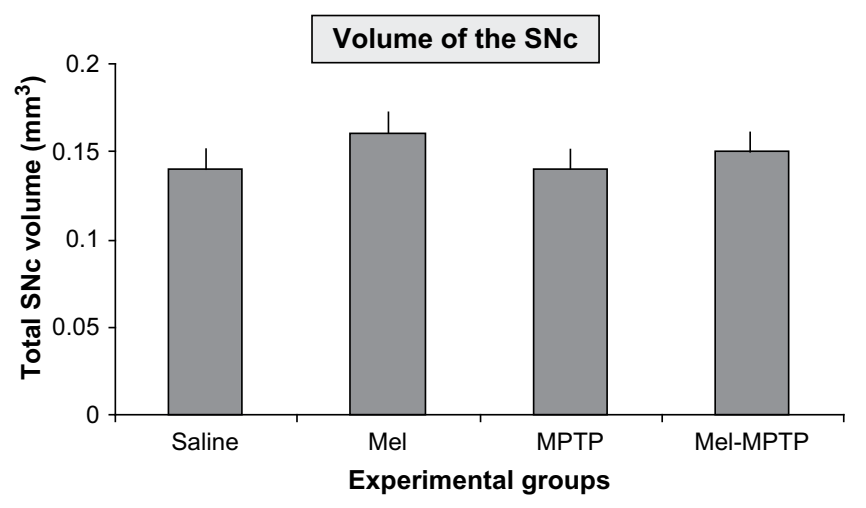

Fig. 5. Graphs showing the volume of the SNc in the four experimental groups used in this study. Columns show the mean \pm 0.01 standard error of the total volume of the $\mathrm{SNc}$ (of right hand side of $\mathrm{TH}+$ sections) in each group. There were no significant differences $(p>0.05)$ in SNc volume between the different groups. There was little variability in the SNc volume in different individual cases of the same group, as indicated by the small standard error bars.

the number of cells found in the Saline and Mel groups (see above; Fig. 6). Hence, compared to the MPTP group, the Mel-MPTP group had $50 \%$ more $\mathrm{TH}^{+}$cells in the SNc. These differences were significant using an ANOVA test $(F=21.7 ; p<0.0001)$. Further, using the multiple comparison test, significant differences (if $q>3.8$ ) were found between the MPTP group and each of the other groups, namely the Saline $(q=7.9 ; p<0.001)$, $\operatorname{Mel}(q=9.6 ; p<0.001)$ and, in particular, the Mel-MPTP ( $q=9.0 ; p<0.001$; see Fig. 6$)$.

In summary, there were $50 \%$ fewer $\mathrm{TH}^{+}$cells in the MPTP group than in the two controls, namely the Saline and Mel groups. The Mel-MPTP group had values similar to the controls and 50\% higher than in the MPTP group. These results indicated that melatonin pretreatment neuroprotects TH expression among SNc cells from the MPTP insult.

\subsubsection{Nissl-stained cells}

The graph in Fig. 7 shows the estimated number of Nissl-stained cells in the SNc of the four experimental groups. In the Saline and Mel groups, the total number of Nissl-stained cells were very similar, being $14,850 \pm 0.011600$ and $15,450 \pm 0.011150$ (Fig. 7).

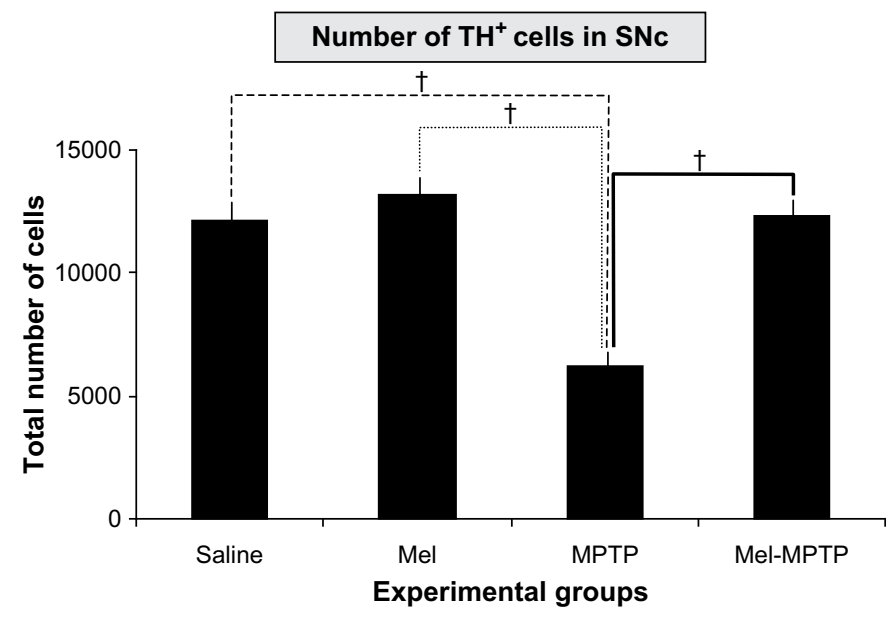

Fig. 6. Graphs showing the number of $\mathrm{TH}^{+}$cells in the SNc in the four experimental groups used in this study. Columns show the mean \pm 0.01 standard error of the total number of SNc cells (of right hand side) in each group. $\dagger$ represents significant difference $(p<0.001)$ in cell number between different groups. There was a $50 \%$ increase in $\mathrm{TH}^{+}$cell number in the Mel-MPTP group compared to the MPTP group. There was little variability in the number of cells in different individual cases of the same group, as indicated by the small standard error bars.
In the MPTP group, the number of Nissl-stained cells was $9900 \pm 0.01600$, indicating $\sim 30 \%$ reduction in SNc cell number from the Saline and Mel groups. For the Mel-MPTP group, the number of Nissl-stained cells was $14,100 \pm 0.011000$, which was similar to the number of cells in the Saline and Mel groups (see above; Fig. 7). Compared to the MPTP group, the Mel-MPTP group had $\sim 30 \%$ more Nissl-stained cells in the SNc. These differences were significant using an ANOVA test $(F=6.7 ; p<0.0001)$. Further, using the multiple comparison test, significant differences (if $q>3.8$ ) were found between the MPTP group and each of the other groups, namely the Saline $(q=4.7 ; p<0.01)$, Mel $(q=5.4$; $p<0.01)$ and the Mel-MPTP $(q=4.3 ; p<0.05$; see Fig. 7$)$.

In summary, there were $\sim 30 \%$ fewer Nissl-stained cells in the MPTP group than in the two controls, namely the Saline and Mel groups. This decrease was within the range, although slightly smaller, than the decrease evident in $\mathrm{TH}^{+}$cell number of the MPTP group. The Mel-MPTP group had values similar to the controls and $\sim 30 \%$ higher than in the MPTP group. Again, this increase was within the range, although slightly smaller, than the increase seen in $\mathrm{TH}^{+}$cell number of the Mel-MPTP group. These results indicated that melatonin pre-treatment not only largely neuroprotected $\mathrm{TH}$ expression among SNc cells, but also helped neuroprotect the cells themselves from MPTP insult.

\section{Discussion}

The most striking finding of this study was that there were more SNc cells in the Mel-MPTP compared to the MPTP group. This indicated that melatonin pre-treatment in MPTP-treated mice offered neuroprotection for these cells. We suggest that this was due to the anti-oxidant properties of melatonin reducing MPTP toxicity. This issue forms the focus of the discussion below. First, a comparison with previous studies is considered.

\subsection{Comparison with previous experimental studies}

To the best of our knowledge, there have only been two other studies exploring the impact of melatonin administration on the

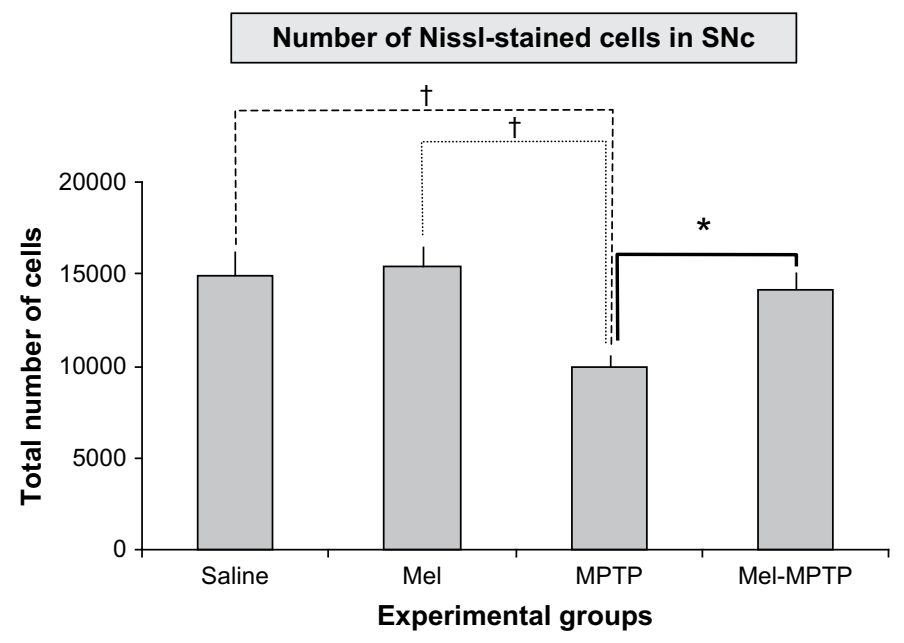

Fig. 7. Graphs showing the number of Nissl-stained cells in the SNc in the four experimental groups used in this study. Columns show the mean \pm 0.01 standard error of the total number of SNc cells (of right hand side) in each group. $\dagger$ represents significant difference at $p<0.01$ in cell number between different groups. * represents significant difference at $p<0.05$ in cell number between different groups. Overall, there was $\sim 30 \%$ increase in Nissl-stained cell number in the Mel-MPTP group compared to the MPTP group. There was little variability in the number of cells in different individual cases of the same group, as indicated by the small standard error bars. 
number of SNc cells after parkinsonian insult [8,9]. These studies used either 6OHDA-lesioned rats [8] or MPTP-treated mice [9] and found similar patterns of cell saving shown in our study (although not from unbiased stereological methods as in present study). It should be noted that there have been many other previous studies examining the effect of melatonin in parkinsonian cases, but these have focussed mainly on changes in dopamine [17], TH [12,13,17], oxidative $[15,16]$ and particular molecular $[3,17]$ activities in the striatum or TH activity in the SNc [18]. There have also been reports on behavioural changes after administration of apomorphine in melatonin-treated 6OHDA-lesioned rats [8,17]. In each case, melatonin increased - most notably - striatal dopamine levels and TH activity and also reduced apomorphine-induced rotational behaviour generated by parkinsonian insult $[13,17]$. In contrast to all the above-mentioned reports, there are two experimental studies that did not find a neuroprotective effect - at least for various dopaminergic markers in the striatum - for melatonin in MPTP-treated mice [10,11]. The reasons for these differences are not clear since the markers analysed and regimes of melatonin and MPTP used by these studies were not too dissimilar to those used by the many positive studies. For example, one of these negative studies had a total melatonin dose of $30 \mathrm{mg} / \mathrm{kg}$ [10], which is well within the range used by the many positive studies $(6-60 \mathrm{mg} / \mathrm{kg}$; see below).

Other substances, in addition to melatonin, have been shown to offer neuroprotection to SNc cells in various animal models of Parkinson disease. For example, caffeine [21], tomato powder [22], glial cell line derived neurotrophic factor [23]) and various dopamine agonists [24], all have been shown to increase dopaminergic cell survival in the SNc or activity in the striatum after parkinsonian insult. Further, neuroprotection in the SNc has also been shown after lesion of deep brain stimulation of various centres that have a glutamatergic inputs to the SNc, for example the subthalamus $[4,25]$. These studies have reported anywhere between 15 and 50\% saving of SNc cells. Despite differences in experimental design, the overall magnitude of neuroprotection found in our present study on melatonin (30-50\%) is comparable - and indeed at the upper end of the scale - to that found in these previous studies. In fact, our results indicated that melatonin potentially neuroprotected all the SNc cells from MPTP insult (see below).

\subsection{Experimental design and limitations of study}

A limitation of this study was that our experimental design did not follow the clinical reality of the parkinsonian condition, where individuals first suffer insult (and SNc cell death) and then have therapeutic intervention. However, our experiments were designed to explore the maximum effect of melatonin on SNc cell number and hence quantify more precisely its neuroprotective effects. This may have been more difficult to achieve if there was a degree of cell death before melatonin administration. Our model was also different to that seen in humans in that it resulted in only $30-50 \%$ death of SNc cells, not as extensive as is normally seen in Parkinson disease when first diagnosed (70-80\% [26]). However, it has been shown that with greater SNc cell death, then there is a smaller magnitude of neuroprotection [4]. Hence, our model was ideal in the sense that it did not generate a massive death of SNc cells, thereby allowing time for therapeutic intervention and a greater scope of possible neuroprotection offered by melatonin [4].

Previous studies have shown that a range of melatonin doses, from 6 to $60 \mathrm{mg} / \mathrm{kg}$, generate neuroprotection of dopaminergic activity in both 6OHDA-lesioned rats and MPTP-treated mice $[8,9,12-18]$ (except for the two studies mentioned above $[10,11])$. In fact, authors have reported no major differences in the magnitude of neuroprotection - in terms of striatal activity - with different dose regimes $[13,15,16]$. In our study, we chose to use a higher dose of melatonin ( $\sim 60 \mathrm{mg} / \mathrm{kg})$, in order to explore its maximum neuroprotective effect. Future studies may explore further whether lower doses of melatonin have a lesser neuroprotective effect in MPTP-treated animals. In 6OHDA-lesioned rats at least, only very small differences have been noted in behaviour and in SNc cell number with different doses of melatonin (6-30 mg/kg [8]).

\subsection{Neuroprotection of SNc cells by melatonin}

There were $30-50 \%$ more SNc cells in the Mel-MPTP compared to the MPTP group, indicating neuroprotection. In fact, we found no significant difference between the number of SNc cells in the controls, namely the Saline and Mel groups, and the Mel-MPTP group, indicating that melatonin treatment neuroprotected nearly all these cells from MPTP toxicity.

Our results did not address directly the mechanism that provided this neuroprotection, but it was likely to be due to the anti-oxidant properties of melatonin. MPTP is thought to impart its toxicity of dopaminergic cells through complex mechanisms. Briefly, once in the brain, MPTP is converted into the active toxin, $\mathrm{MPP}^{+}$, that then concentrates within the mitochondria. There, it inhibits the activity of complex 1 of the electron transport chain leading ultimately to ATP depletion and oxygen free radical production; this process causes much damage to cell proteins, including TH [27]. Melatonin, with its known potent free radical scavenger properties and ability to increase both mitochondrial respiration and ATP synthesis, presumably helped the dopaminergic cells to become more resistant to the above-mentioned damaging effects of MPTP [16]. Hence, and in relation to our findings in this study, the overall numbers of SNc cells in the Mel-MPTP group were much higher than in the MPTP group.

\subsection{Functional and true neuroprotection}

The present finding that there were both more $\mathrm{TH}^{+}$and Nisslstained cells in the Mel-MPTP compared to the MPTP group, indicated that there was both a functional and true neuroprotection by melatonin. In other words, there was an actual increase in SNc cell survival and not just a rescue of the dopaminergic phenotype. This result confirms the findings of some studies [4], but not of another [28]. One may argue that it is difficult to compare the findings of these previous studies and ours, mainly because of the different experimental designs. Each study, for example, had a different means of cell labelling (e.g., tracer-labelled vs Nissl-stained) and used different animal models of the disease (e.g., 6OHDA-lesioned rats vs MPTP-treated mice or monkeys). Perhaps a more insightful comparison on the issue of functional vs true neuroprotection may come from a study that incorporates a common experimental design.

Our results indicated also that in each group, there were always more Nissl-stained than $\mathrm{TH}^{+}$cells in the SNc. This finding may have reflected several - not necessarily mutually exclusive - features. First, a proportion of SNc cells were "sick", due to MPTP toxicity, and not expressed $\mathrm{TH}$. These cells, although functionally impaired, were still Nissl-stained. It has been shown that toxins such as MPTP have initial impact on transmitter phenotype (i.e., TH) and then, after a longer period, on the overall cell itself, manifesting in death [29]. Second, there were different patterns of SNc cell labelling by the two techniques used, namely immunochemistry and Nissl staining. For instance, immunochemistry perhaps did not label all the cells in the section (i.e., those in the middle) because of limited antibody penetration. Nissl staining has been shown to label all the cells across the entire thickness of the section [30]. Third, there may have been differences in defining the SNc border using the two methods; the borders with Nissl staining were not as clear and some more cells (outside the SNc) may have been included in these 
counts. Finally, TH immunochemistry did not reveal a distinct population of non- $\mathrm{TH}^{+}$cells in the SNc; these cells would have been revealed by Nissl staining [31]. Taken together, these features were likely to have contributed to the finding that there were fewer $\mathrm{TH}^{+}$ than Nissl-stained cells in all the experimental groups of this study.

\section{Conclusion and clinical relevance}

Our results indicated that melatonin administration in MPTPtreated mice potentially saved all SNc cells from death. We suggest that the mechanism underlying this saving is the anti-oxidant properties of melatonin. Future studies may explore whether this suggestion is, in fact, the case.

There are several conflicting reports on the validity of using melatonin therapy in a clinical setting. On the one hand, melatonin treatment has been reported to have little symptomatic relief for patients [32] and in some cases, may even exacerbate the symptoms by inhibiting dopamine production by cells in the SNc [33]. Further, some studies have indicated that melatonin levels are not necessarily low in parkinsonian patients [34]. Finally, it has been reported that melatonin administration may have many sideeffects on patients [35]. On the other hand, however, previous work has in fact reported symptomatic relief in parkinsonian cases with melatonin therapy [36]; further, that dysfunction of the pineal gland - the major site of melatonin production - plays an important role in the generation of parkinsonism [37]. In this context, our results - albeit on an animal model of Parkinson disease - provide some encouragement that perhaps at the right dose, melatonin may be used to help save or prevent the death of SNc cells in Parkinson disease. Future studies may explore this issue further.

\section{Acknowledgements}

We are forever grateful to Tenix Corp and Salteri family for the generous funding of the laboratory. We thank Sharon Spana for technical assistance.

\section{References}

[1] Rinne JO. Nigral degeneration in Parkinson's disease. Mov Disord 1993; 8(Suppl. 1):S31-5.

[2] Blandini F, Nappi G, Tassorelli C, Martignoni E. Functional changes of the basal ganglia circuitry in Parkinson's disease. Prog Neurobiol 2000;62:63-88.

[3] Jankovic J. Motor fluctuations and dyskinesias in Parkinson's disease: clinical manifestations. Mov Disord 2005;20:S11-6.

[4] Wallace BA, Ashkan K, Heise CE, Foote KD, Torres N, Mitrofanis J, et al. Survival of midbrain dopaminergic cells after lesion or deep brain stimulation of the subthalamic nucleus in MPTP-treated monkeys. Brain 2007;130:2129-45.

[5] Reiter RJ. Neuroendocrinology of melatonin. In: Miles A, Philbrick DRS, Thompson C, editors. Melatonin - clinical perspectives. Oxford: Oxford University Press; 1988. p. 1-42.

[6] Ianas O, Olinescu R, Badescu I. Melatonin involvement in oxidative processes. Endocrinologie 1991;29:147-53.

[7] Hara M, Yoshida M, Nishijima H, Yokosuka M, ligo M, Ohtani-Kaneko R, et al. Melatonin a pineal secretory product with antioxidant properties protects against cisplatin-induced nephrotoxicity in rats. J Pineal Res 2001;30:129-38.

[8] Kim YS, Joo WS, Jin BK, Cho YH, Baik HH, Park CW. Melatonin protects 6OHDAinduced neuronal death of nigrostriatal dopaminergic system. Neuroreport 1998;13:2387-90.

[9] Antolın I, Mayo JC, Sainz RM, de los Angeles del Brıo M, Herrera F, Martın V, et al. Protective effect of melatonin in a chronic experimental model of Parkinson's disease. Brain Res 2002;943:163-73.

[10] Itzhak Y, Martin JL, Dean Black M, Ali SF. Effect of melatonin on methamphetamine- and 1-methyl-4-phenyl-1,2,3,6-tetrahydropyridine-induced dopaminergic neurotoxicity and methamphetamine-induced behavioural sensitization. Neuropharmacology 1998;37:781-91.

[11] Morgan WW, Nelson JF. Chronic administration of pharmacological levels of melatonin does not ameliorate the MPTP-induced degeneration of the nigrostriatal pathway. Brain Res 2001;921:115-21.

[12] Acuna-Castroviejo D, Coto-Montes A, Gaia Monti M, Ortiz GG, Reiter RJ Melatonin is protective against MPTP-induced striatal and hippocampal lesions. Life Sci 1997;60:23-9.

[13] Joo WS, Jin BK, Park CW, Maeng SH, Kim YS. Melatonin increases striatal dopaminergic function in 6-OHDA-lesioned rats. Neuropharmacology 1998;9:4123-6.

[14] Mayo JC, Sainz RM, Uria H, Antolin I, Esteban MM, Rodriguez C. Melatonin prevents apoptosis induced by 6-hydroxydopamine in neuronal cells: implications for Parkinson disease. J Pineal Res 1998;24:179-92.

[15] Dabbeni-Sala F, Di Santo S, Franceschini D, Skaper SD, Giusti P. Melatonin protects against 6OHDA-induced neurotoxicity in rats: a role for mitochondrial complex I activity. FASEB J 2001;15:164-70.

[16] Thomas B, Mohanakumar KP. Melatonin protects against oxidative stress caused by 1-methyl-4-phenyl-1236-tetrahydropyridine in the mouse nigrostriatum. J Pineal Res 2004;36:25-32.

[17] Sharma R, McMillan CR, Tenn CC, Niles LP. Physiological neuroprotection by melatonin in a 6-hydroxydopamine model of Parkinson disease. Brain Res 2006;1068:230-6.

[18] Jin BK, Shin DY, Jeong MY, Gwag MR, Baik HW, Yoon KS, et al. Melatonin protects nigral dopaminergic neurons from 1-methyl-4-phenyl-pyridinium $(\mathrm{MPP}+)$ neurotoxicity in rats. Neurosci Lett 1998;245:61-4.

[19] Paxinos G, Franklin KBJ. The mouse brain in stereotaxic coordinates. 2nd ed San Diego, CA: Academic Press; 2001.

[20] Aguirre JA, Andbjer B, Gonzalez-Baron S, Hansson A, Stromberg I, Agnati LF, et al. Group I mGluR antagonist AIDA protects nigral DA cells from MPTPinduced injury. Neuroreport 2001;12:2615-7.

[21] Vasconcelos-Aguiar LM, Nobre Jr HV, Macêdo DS, Oliveira AA, Freitas RM Vasconcelos SM, et al. Neuroprotective effects of caffeine in the model of 6 hydroxydopamine lesion in rats. Pharmacol Biochem Behav 2006;84:415-9.

[22] Suganuma H, Hirano T, Arimoto Y, Inakuma T. Effect of tomato intake on striatal monoamine level in a mouse model of experimental Parkinson's disease. J Nutr Sci Vitaminol 2002;48:251-4.

[23] Georgievska B, Kirik D, Bjorklund A. Aberrant sprouting and downregulation of tyrosine hydroxylase in lesioned nigrostriatal dopamine neurons induced by long-lasting overexpression of glial cell line derived neurotrophic factor in the striatum by lentiviral gene transfer. Exp Neurol 2002;177:461-74.

[24] Riederer P, Gille G, Müller T, Przuntek H, Reichmann H, Riess O, et al. Practica importance of neuroprotection in Parkinson's disease. J Neurol 2002;249:53-6.

[25] Piallat B, Benazzouz A, Benabid AL. Subthalamic nucleus lesion in rats prevents dopaminergic nigral neuron degeneration after striatal 6-OHDA injection: behavioural and immunohistochemical studies. Eur J Neurosci 1996;8:1408-14.

[26] Ashkan K, Wallace B, Bell BA, Benabid AL. Deep brain stimulation of the subthalamic nucleus in Parkinson's disease 1993-2003: where are we 10 years on? Br J Neurosurg 2004; 18:19-34.

[27] Przedborski S, Jackson-Lewis V, Djaldetti R, Liberatore G, Vila M, Vukosavic S, et al. The parkinsonian toxin MPTP: action and mechanism. Restor Neurol Neurosci 2000;16:135-42.

[28] Paul G, Meissner W, Rein S, Harnack D, Winter C, Hosmann K, et al. Removal of the subthalamic nucleus protects dopaminergic phenotype but not cell survival in a rat model of Parkinson's disease. Exp Neurol 2004;185:272-80.

[29] Bjorklund A, Rosenblad C, Winkler C, Kirik D. Studies on neuroprotective and regenerative effects of GDNF in a partial lesion model of Parkinson's disease. Neurobiol Dis 1997;4:186-200.

[30] Mitrofanis J. Calbindin immunoreactivity in a subset of cat thalamic reticular neurones. J Neurocytol 1992;21:495-505.

[31] Fallon JH, Loughlin SE. Monoamine innervation of the forebrain: collaterilisation. Brain Res Bull 1982;9:295-307.

[32] Shaw KM, Stern GM, Sandler M. Melatonin and parkinsonism. Lancet 1973;1:271.

[33] Zisapel N. Melatonin-dopamine interactions: from basic neurochemistry to a clinical setting. Cell Mol Neurobiol 2001;21:605-16.

[34] Bordet R, Devos D, Brique S, Touitou Y, Guieu JD, Libersa C, et al. Study of circadian melatonin secretion pattern at different stages of Parkinson's disease. Clin Neuropharmacology 2003;26:65-72.

[35] Guardiola-Lemaitre B. Toxicology of melatonin. J Biol Rhythms 1997;12: 697-706.

[36] Anton-Tay F, Diaz JL, Fernandez-Guardiola A. On the effect of melatonin upon human brain. Its possible therapeutic implications. Life Sci I 1971;10:841-50.

[37] Willis GL. The role of ML-23 and other melatonin analogues in the treatment and management of Parkinson's disease. Drug News Perspect 2005;8:437-44. 